\title{
6-jähriges Mädchen mit suprapatellarer, medial betonter Knieschwellung
}

Bei einer Knieschwellung im Kindesalter sind viele Differenzialdiagnosen zu berücksichtigen. Die häufigsten Ursachen sind ein Trauma, eine Infektion oder eine rheumatische Erkrankung. Ein Tumor oder eine Gefäßmalformation sollten bei unilateraler Schwellung ebenfalls in Betracht gezogen werden. Um die Palette der möglichen Diagnosen deutlich einzuengen, hilft bereits eine ausführliche Anamnese mit klinischer Untersuchung gefolgt von Laborwerten und Bildgebung.

Wir berichten über ein 6-jähriges, immunkompetentes Mädchen, welches mit seit 3 Tagen bestehender Schwellung und Schmerzen des rechten Kniegelenkes zunächst bei den Kollegen der Kinderchirurgie vorgestellt wurde. Da in der Anamnese kein Trauma und kein Hinweis auf Verletzung bestanden, erfolgte die Weiterleitung zur weiteren Abklärung in unsere Klinik. Bei der Untersuchung zeigte sich eine suprapatellare Schwellung rechts mit leichter Überwärmung sowie eine schmerzhafte Bewegungseinschränkung ( $\mathbf{A} \mathbf{b} \mathbf{b} . \mathbf{1}$ ). Bemerkenswert waren die mediale Betonung der Schwellung und der Palpationsbefund (sehr weich, nicht fluktuierend). Laborchemisch zeigten sich keine relevanten Inflammationsparameter, die BSG lag bei $8 \mathrm{~mm} / \mathrm{h}$, sodass eine Infektion oder starke Entzündung unwahrscheinlich waren. Das konventionelle Röntgenbild des rechten Knies war unauffällig. Sonografisch zeigte sich ein „verdickter“ M. vastus medialis des M. quadriceps femoris (-Abb. 2) mit verstärkter Durchblutung ( Abb.3). Damit lag der Verdacht auf eine vaskuläre Malformation nahe.

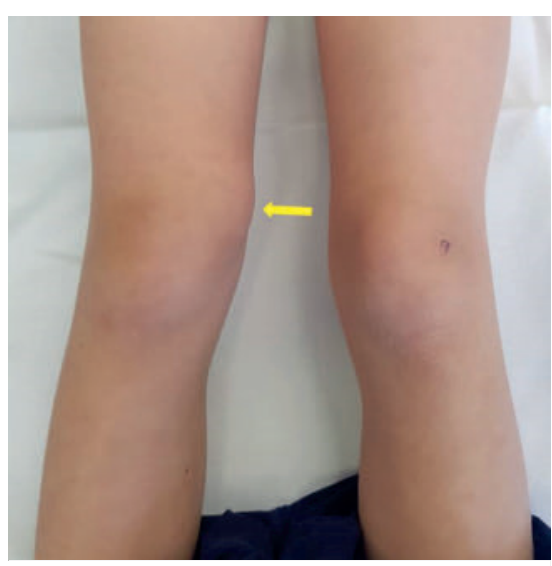

- Abb. 1 Suprapatellare, medial betonte Knieschwellung.

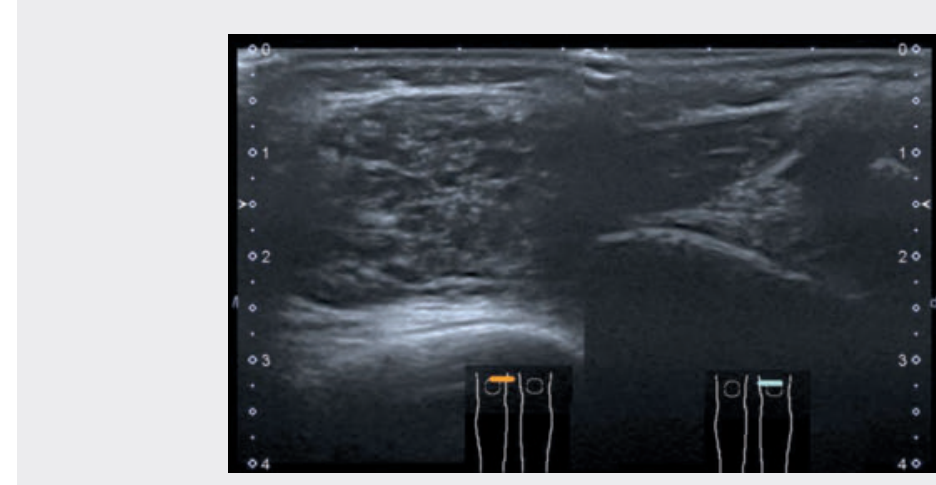

- Abb. 2 Sonografie: „verdickter“ M. vastus medialis des M. quadriceps femoris rechts.

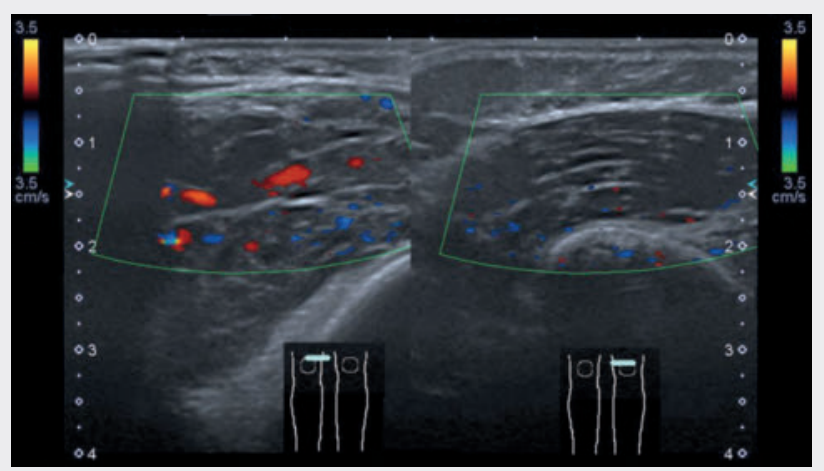

- Abb.3 Gleiche Region in der Doppler-Sonografie mit verstärkter Durchblutung.

Die Patientin wurde nach ambulanter Betreuung mit symptomatischer Naproxen-Therapie nach Hause entlassen, ein ambulantes MRT zur weiteren Einordnung des sonografischen Befundes war geplant. Im PD-Kontrast mit Fettsättigung lag am distalen Femur medial, teils im M. vastus medialis, teils juxtaartikulär im suprapatellaren Recessus im periossalen Fettgewebe eine ca. 36×35×19 mm messende Raumforderung ( $\triangleright$ Abb. 4 bis $\triangleright$ Abb. 6), passend zu einer juxtaartikulären venösen Malformation. Das mediale femoropatellare Ligament wird von dieser Struktur umgeben ( Abb. 7).

Venöse Malformationen sind benigne angeborene, vaskuläre Anomalien mit langsamem Fluss, bestehend aus erweiterten venösen Kanälen mit dünner Wand. Intraartikuläre venöse Malformationen des Knies sind ein seltener Subtyp der venösen Malformationen. Zu den häufigsten Symptomen gehören unspezifische Schmerzen und

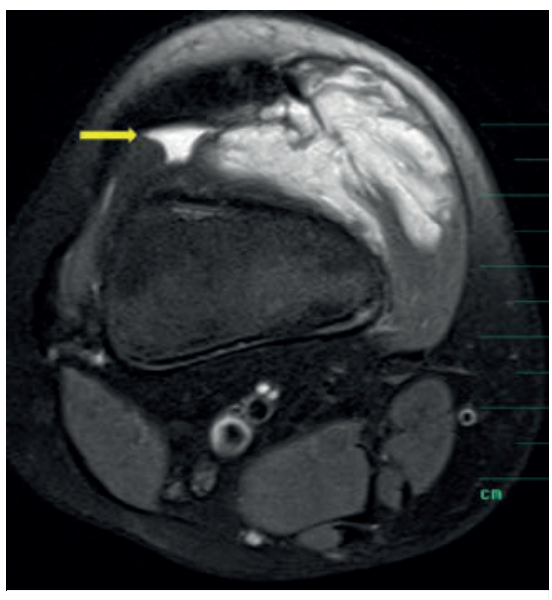

- Abb. 4 MRT axial PD-gewichtete TSE mit Fettsuppression (gelber Pfeil = minimaler Kniegelenkserguss).

Gelenkschwellung, begleitet von einer Bewegungseinschränkung, welche episodisch auftreten können. Bei anhaltenden Symptomen kann eine sekundäre Muskelatrophie vorhanden sein. Äußere Zeichen wie 


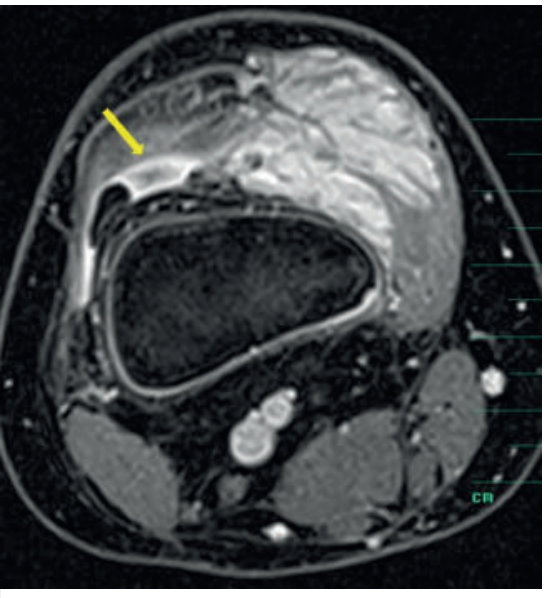

Abb.5 MRT T1-Wichtung axial nach KM: juxtaartikuläre, synoviale Slow-FlowMalformation (gelber Pfeil = Kniegelenkserguss und wenig Synovialitis).

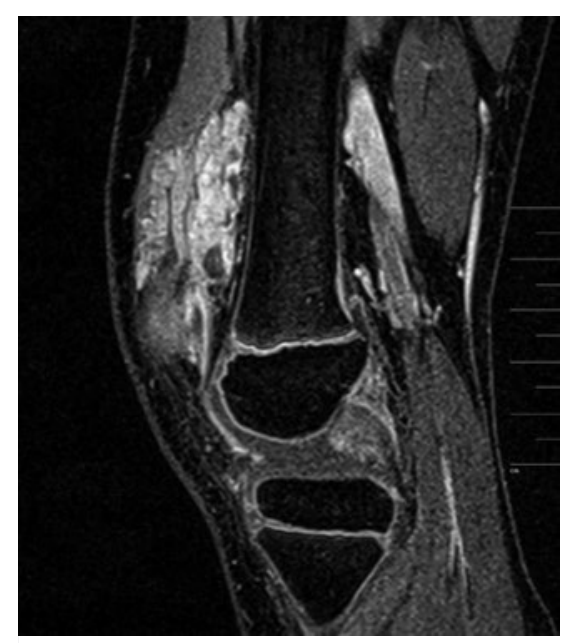

- Abb. 6 MRT T1-Wichtung mit Fettsuppression nach KM sagittal.

Varizen oder kapillare Malformationen der Haut können auf venöse Malformationen hinweisen. Jedoch können kutane Veränderungen fehlen, und die Symptomatik kann häufigeren Diagnosen wie juvenile idiopathische Arthritis, Trauma oder einem Tumor ähneln. Die rasche Diagnose einer intraartikulären venösen Malformation des Knies ist essenziell, um eine Gelenkblutung und sekundären Knorpelschaden zu verhindern. Deswegen sind die Kenntnis der Klinik von venösen Malformationen und die Vertrautheit mit MRT-Besonderheiten dieser Erkrankung wichtig und nützlich. Mittels MRT gelingt in der Regel auch die Abgrenzung zu anderen wichtigen Differenzialdiagnosen ( Tab. 1).

\Tab. 1 MRT-Charakteristika dreier wichtiger Synovialläsionen.

\begin{tabular}{|l|l|l|l|}
\hline & Venöse Malformation & PVNS & Synovialsarkom \\
\hline & +++ & +++ & +++ \\
\hline Diskrete Masse & ++ & + & ++ \\
\hline Scharfe Begrenzung & +++ & ++ & +++ \\
\hline Anreicherung & +++ & $(-)$ & + \\
\hline Extraartikuläre Ausdehnung & - & + & - \\
\hline Extraartikuläres Ödem & +++ & ++ & + \\
\hline Knöcherne Beteiligung & - & ++ & - \\
\hline Knochenödem & + & ++ & +++ \\
\hline Gelenkerguss & & & + \\
\hline
\end{tabular}

PVNS = pigmentierte villonoduläre Synovialitis

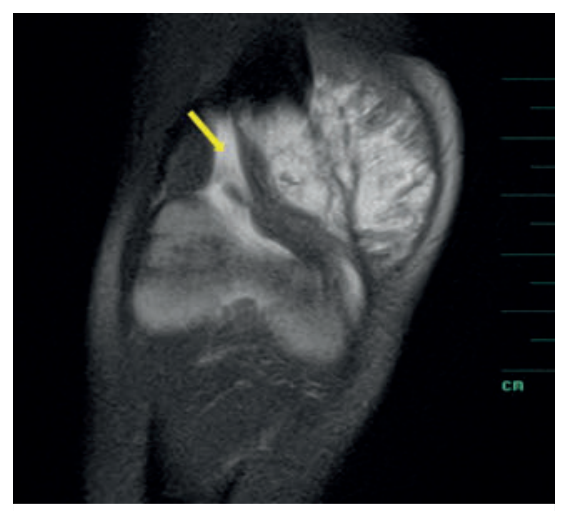

- Abb. 7 MRT PD-Wichtung coronar mit Fettsuppression, ca. $36 \times 35 \times 19 \mathrm{~mm}$ große venöse Slow-Flow-Malformation (gelber Pfeil = imprimierter Erguss im Recessus suprapatellaris).

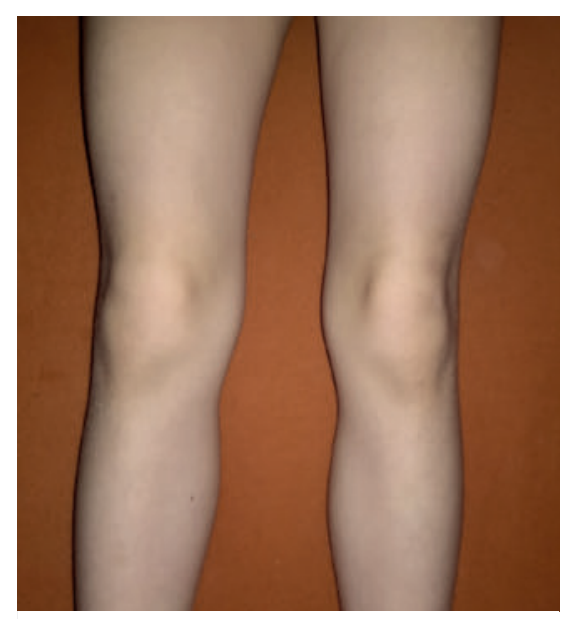

- Abb. 8 Spontane Regression der Knieschwellung 2 Monate später.
Zur diagnostischen Bildgebung bei unspezifischen (unilateralen) Knieschmerzen gehört ein Röntgen, das bei intraartikulären venösen Malformationen oft unauffällig ist. Das MRT-Protokoll für vaskuläre Anomalien sollte T1- und T2-Turbo-Spin-Echo-Sequenzen mit und ohne Fettsättigung einschließen sowie fettgesättigte T-1 Sequenzen nach Kontrastmittelgabe.

Im Kontrast-MRT erscheinen venöse Malformationen wie tubuläre Strukturen mit hoher Signalintensität in T2-gewichteten Sequenzen und mit mittlerer bis niedriger Signalintensität in T1-gewichteten Sequenzen. Die Morphologie der venösen Malformationen ist am besten in T2gewichteten, fettgesättigten Sequenzen zu beurteilen und variiert von gelappten, clusterartigen Strukturen bis zu diffus infiltrierenden Phlebektasien. Die T2-Turbo-Spin-
Echo-Sequenz in sagittaler Schnittführung ist insbesondere hilfreich in der Beurteilung einer exakten Anatomie der Malformation und in einer möglichen Synovialbeteiligung im interkondylären Raum oder im suprapatellaren Rezessus. Kontrastmittelgabe hilft, das Gewebe besser zu charakterisieren und unterstützt den Nachweis der vaskulären Genese einer Läsion. Da venöse Malformationen an die systemische Zirkulation per Kapillaren gebunden sind, füllen sie sich mit Kontrastmittel auf.

Bei unserer Patientin war die suprapatellare Schwellung spontan wieder rückläufig ( $\triangleright$ Abb. 8). Dies bedeutet aber keine Spontanheilung, sondern ist Ausdruck des unterschiedlichen Füllungszustandes der venösen Malformation. Der interventionelle, gefäßchirurgische Eingriff ist geplant. 


\section{ZUSAMMENFASSUNG}

In unserem Fall wurde die venöse Malformation trotz ihrer Seltenheit rasch festgestellt. Wegweisend für die Diagnose waren die suprapatellare Lokalisation der Schwellung mit medialer Betonung, fehlende Entzündungsparameter im Blut, sowie die Sonografie. Mit dem Kontrastmittel-MRT konnte der Verdacht einer vaskulären Malformation bestätigt und auf eine juxtaartikuläre venöse Malformation präzisiert werden.

\section{Interessenkonflikt}

Die Autorinnen/Autoren geben an, dass kein Interessenkonflikt besteht.

\section{Einhaltung ethischer Richtlinien}

Die Autorinnen/Autoren bestätigen, dass eine schriftliche Einwilligung der Eltern der Patientin zur Publikation dieses Fallberichts vorliegt.

\section{Danksagung}

Die Autoren danken dem Kinderradiologen K. Jablonka herzlich für die Beratung bei der Auswahl sowie für die Befundung der Sonografie- und MRT-Bilder.

\section{Autorinnen/Autoren}

\section{J. Weise, F. Weller-Heinemann}

Prof.-Hess-Kinderklinik Bremen

Gesundheit Nord gGmbH

\section{Korrespondenzadresse}

\section{F. Weller-Heinemann}

OA Kinder- und Jugendrheumatologie Prof. Hess Kinderklink Bremen

Gesundheit Nord gGmbH

Friedrich Karl Straße, 28177 Bremen

Deutschland

frank.weller@klinikum-bremen-mitte.de
Literatur

[1] Mattila K, Aronniemi J, Salminen P et al. Intraarticular venous malformation of the knee in children: magnetic resonance imaging findings and significance of synovial involvement. Pediatric Radiology 2020; 50: 509-515

[2] Sheldon P, Forrester D, Learch T. Imaging of Intraarticular masses. RadioGraphics 2005; 25: $105-119$

[3] Kan J, Hernanz-Schulman M, Damon B et al. MRI features of three paediatric intraarticular synovial lesions: a comparative study. Clinical Radiology 2008; 63: 805-812

[4] Tsai A, Chaudry G, Spencer S et al. Misdiagnosis of knee venous malformation as juvenile idiopathic arthritis. J Pediatr Orthop 2011; 31: 683-690

[5] Brisse H, Orbach D, Klijanienko J et al. Imaging and diagnostic strategy of soft tissue tumors in children. Eur Radiol 2006; 16: 1147-1164

\section{Bibliografie}

DOI https://doi.org/10.1055/a-1337-9249 arthritis + rheuma 2021; 41: 53-55 (c) 2021. Thieme. All rights reserved. Georg Thieme Verlag KG, Rüdigerstraße 14, 70469 Stuttgart, Germany ISSN 0176-5167 